\title{
Compton scattering of quasi-real virtual photons at LEP
}

\section{L3 Collaboration}

P. Achard ${ }^{\mathrm{t}}$, O. Adriani ${ }^{\mathrm{q}}$, M. Aguilar-Benitez ${ }^{\mathrm{y}}, \mathrm{J}_{\text {. Alcaraz }}^{\mathrm{y}}$, G. Alemanni ${ }^{\mathrm{w}}$, J. Allaby ${ }^{\mathrm{r}}$, A. Aloisio $^{\text {ac }}$, M.G. Alviggi ${ }^{\text {ac }}$, H. Anderhub ${ }^{\text {aw }}$, V.P. Andreev ${ }^{\text {f,ah }}$, F. Anselmo ${ }^{\text {h }}$,

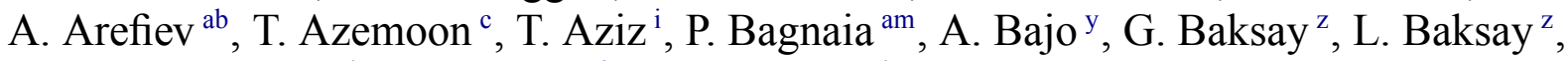

S.V. Baldew ${ }^{\mathrm{b}}$, S. Banerjee ${ }^{\mathrm{i}}$, Sw. Banerjee ${ }^{\mathrm{d}}$, A. Barczyk ${ }^{\mathrm{aw}, \mathrm{au}}$, R. Barillère ${ }^{\mathrm{r}}$, P. Bartalini ${ }^{\text {w }}$, M. Basile ${ }^{\text {h }}$, N. Batalova ${ }^{\text {at }}$, R. Battiston ${ }^{\text {ag }}$, A. Bay ${ }^{\text {w }}$, F. Becattini ${ }^{\text {q }}$, U. Becker ${ }^{\mathrm{m}}$, F. Behner ${ }^{\text {aw }}$, L. Bellucci ${ }^{\mathrm{q}}$, R. Berbeco ${ }^{\mathrm{c}}$, J. Berdugo ${ }^{\mathrm{y}}$, P. Berges ${ }^{\mathrm{m}}$, B. Bertucci ${ }^{\text {ag }}$, B.L. Betev ${ }^{\text {aw }}$, M. Biasini ${ }^{\text {ag }}$, M. Biglietti ${ }^{\text {ac }}$, A. Biland ${ }^{\text {aw }}$, J.J. Blaising ${ }^{\text {d, }}$ S.C. Blyth ${ }^{\text {ai }}$, G.J. Bobbink ${ }^{\text {b }}$, A. Böhm ${ }^{\text {a }}$, L. Boldizsar ${ }^{1}$, B. Borgia ${ }^{\text {am }}$, S. Bottai ${ }^{\mathrm{q}}$, D. Bourilkov ${ }^{\text {aw }}$, M. Bourquin ${ }^{\mathrm{t}}$, S. Braccini ${ }^{\mathrm{t}}$, J.G. Branson ${ }^{\text {ao }}$, F. Brochu ${ }^{\mathrm{d}}$, J.D. Burger ${ }^{\mathrm{m}}$, W.J. Burger ${ }^{\mathrm{ag}}$, X.D. Cai ${ }^{\mathrm{m}}$, M. Capell ${ }^{\mathrm{m}}$, G. Cara Romeo ${ }^{\mathrm{h}}$, G. Carlino ${ }^{\mathrm{ac}}$, A. Cartacci ${ }^{\mathrm{q}}$, J. Casaus $^{\mathrm{y}}$, F. Cavallari ${ }^{\mathrm{am}}$, N. Cavallo ${ }^{\text {aj }}$, C. Cecchi ${ }^{\mathrm{ag}}$, M. Cerrada ${ }^{\mathrm{y}}$, M. Chamizo ${ }^{\mathrm{t}}$, Y.H. Chang ${ }^{\text {ar }}$, M. Chemarin ${ }^{\mathrm{x}}$, A. Chen ${ }^{\text {ar }}$, G. Chen ${ }^{\mathrm{g}}$, G.M. Chen ${ }^{\mathrm{g}}$, H.F. Chen ${ }^{\mathrm{v}}$, H.S. Chen ${ }^{\mathrm{g}}$, G. Chiefari ${ }^{\text {ac }}$, L. Cifarelli ${ }^{\text {an }}$, F. Cindolo ${ }^{\text {h }}$, I. Clare ${ }^{\mathrm{m}}$, R. Clare ${ }^{\text {al }}$, G. Coignet ${ }^{\mathrm{d}}$, N. Colino ${ }^{\mathrm{y}}$, S. Costantini ${ }^{\mathrm{am}}$, B. de la Cruz ${ }^{\mathrm{y}}$, S. Cucciarelli ${ }^{\mathrm{ag}}$, R. de Asmundis ${ }^{\text {ac }}$, P. Déglon ${ }^{t}$, J. Debreczeni ${ }^{1}$, A. Degré ${ }^{\mathrm{d}}$, K. Dehmelt ${ }^{\mathrm{z}}$, K. Deiters ${ }^{\text {au }}$, D. della Volpe ${ }^{\text {ac }}$, E. Delmeire ${ }^{t}$, P. Denes ${ }^{\text {ak }}$, F. DeNotaristefani ${ }^{a m}$, A. De Salvo ${ }^{\text {aw }}$, M. Diemoz ${ }^{\text {am }}$, M. Dierckxsens ${ }^{\mathrm{b}}$, C. Dionisi ${ }^{\text {am }}$, M. Dittmar ${ }^{a w}$, A. Doria ${ }^{\text {ac }}$, M.T. Dova ${ }^{\mathrm{j}, 1}$, D. Duchesneau ${ }^{d}$, M. Duda a ${ }^{a}$ B. Echenard ${ }^{t}$, A. Eline ${ }^{r}$, A. El Hage ${ }^{a}$, H. El Mamouni ${ }^{x}$, A. Engler ${ }^{\text {ai }}$, F.J. Eppling ${ }^{\mathrm{m}}$, P. Extermann ${ }^{\mathrm{t}}$, M.A. Falagan ${ }^{\mathrm{y}}$, S. Falciano ${ }^{\mathrm{am}}$, A. Favara ${ }^{\text {af }}$, J. Fay ${ }^{x}$, O. Fedin ${ }^{\text {ah }}$, M. Felcini ${ }^{\text {aw }}$, T. Ferguson ${ }^{\text {ai }}$, H. Fesefeldt ${ }^{\text {a }}$, E. Fiandrini ${ }^{\text {ag }}$, J.H. Field ${ }^{\mathrm{t}}$, F. Filthaut ${ }^{\mathrm{ae}}$, P.H. Fisher ${ }^{\mathrm{m}}$, W. Fisher ${ }^{\text {ak }}$, I. Fisk ${ }^{\mathrm{ao}}$, G. Forconi ${ }^{\mathrm{m}}$, K. Freudenreich ${ }^{\text {aw }}$, C. Furetta ${ }^{\text {aa }}$, Yu. Galaktionov ${ }^{\text {ab, }}{ }^{\text {, S.N. Ganguli }}{ }^{\mathrm{i}}$, P. Garcia-Abia ${ }^{\mathrm{y}}$, M. Gataullin af ${ }^{\text {, S. Gentile }}{ }^{\text {am }}$, S. Giagu ${ }^{\text {am }}$, Z.F. Gong ${ }^{v}$, G. Grenier ${ }^{\mathrm{x}}$, O. Grimm ${ }^{\mathrm{aw}}$, M.W. Gruenewald ${ }^{\mathrm{p}}$, M. Guida ${ }^{\text {an }}$, V.K. Gupta ${ }^{\mathrm{ak}}$, A. Gurtu ${ }^{\mathrm{i}}$, L.J. Gutay ${ }^{\text {at }}$, D. Haas ${ }^{\mathrm{e}}$, D. Hatzifotiadou ${ }^{\text {h}}$, T. Hebbeker ${ }^{\text {a }}$, A. Hervé ${ }^{r}$, J. Hirschfelder ${ }^{\text {ai }}$, H. Hofer ${ }^{\text {aw }}$, M. Hohlmann ${ }^{\text {z }}$, G. Holzner ${ }^{\text {aw }}$, S.R. Hou ${ }^{\text {ar }}$, B.N. Jin ${ }^{\mathrm{g}}$, P. Jindal ${ }^{\mathrm{n}}$, L.W. Jones ${ }^{\mathrm{c}}$, P. de Jong ${ }^{\text {b }}$, I. Josa-Mutuberría ${ }^{y}$, M. Kaur ${ }^{\mathrm{n}}$, M.N. Kienzle-Focacci ${ }^{\mathrm{t}}$, J.K. Kim ${ }^{\text {aq }}$, 
J. Kirkby ${ }^{\mathrm{r}}$, W. Kittel ${ }^{\text {ae }}$, A. Klimentov ${ }^{\mathrm{m}, \mathrm{ab}}$, A.C. König ${ }^{\text {ae }}$, M. Kopal ${ }^{\text {at }}$, V. Koutsenko ${ }^{\mathrm{m}, \mathrm{ab}}$,

M. Kräber ${ }^{\text {aw }}$, R.W. Kraemer ai, A. Krüger ${ }^{\text {av }}$, A. Kunin ${ }^{\text {m }}$, P. Ladron de Guevara ${ }^{y}$,

I. Laktineh ${ }^{\mathrm{x}}$, G. Landi ${ }^{\mathrm{q}}$, M. Lebeau ${ }^{\mathrm{r}}$, A. Lebedev ${ }^{\mathrm{m}}$, P. Lebrun ${ }^{\mathrm{x}}$, P. Lecomte ${ }^{\text {aw }}$,

P. Lecoq ${ }^{r}$, P. Le Coultre ${ }^{\text {aw }}$, J.M. Le Goff ${ }^{\mathrm{r}}$, R. Leiste ${ }^{\text {av }}$, M. Levtchenko ${ }^{\text {aa }}$, P. Levtchenko ${ }^{\text {ah }}$, C. Li $^{\text {v }}$, S. Likhoded ${ }^{\text {av }}$, C.H. Lin ${ }^{\text {ar }}$, W.T. Lin ${ }^{\text {ar }}$, F.L. Linde ${ }^{\text {b }}$, L. Lista ${ }^{\text {ac }}$, Z.A. Liu ${ }^{\mathrm{g}}$, W. Lohmann ${ }^{\text {av }}$, E. Longo ${ }^{\text {am }}$, Y.S. Lu ${ }^{\mathrm{g}}$, C. Luci ${ }^{\text {am }}$, L. Luminari ${ }^{\mathrm{am}}$, W. Lustermann ${ }^{\text {aw }}$, W.G. Ma ${ }^{\mathrm{v}}$, L. Malgeri ${ }^{\mathrm{r}}$, A. Malinin ${ }^{\mathrm{ab}}$, C. Maña ${ }^{\mathrm{y}}$, J. Mans ${ }^{\text {ak }}$, J.P. Martin ${ }^{\mathrm{x}}$, F. Marzano ${ }^{\mathrm{am}}$, K. Mazumdar ${ }^{\mathrm{i}}$, R.R. McNeil ${ }^{\mathrm{f}}$, S. Mele ${ }^{\mathrm{r}, \mathrm{ac}}$, L. Merola ${ }^{\text {ac }}$, M. Meschini ${ }^{\mathrm{q}}$, W.J. Metzger ${ }^{\text {ae }}$, A. Mihul ${ }^{\mathrm{k}}$, H. Milcent ${ }^{\mathrm{r}}$, G. Mirabelli ${ }^{\text {am }}$, J. Mnich ${ }^{\mathrm{a}}$, G.B. Mohanty ${ }^{\mathrm{i}}$, G.S. Muanza ${ }^{\mathrm{x}}$, A.J.M. Muijs ${ }^{\mathrm{b}}$, B. Musicar ${ }^{\mathrm{ao}}$, M. Musy ${ }^{\mathrm{am}}, \mathrm{S}$ S. Nagy ${ }^{\mathrm{o}}$,

S. Natale ${ }^{t}$, M. Napolitano ${ }^{\text {ac }}$, F. Nessi-Tedaldi ${ }^{\text {aw }}$, H. Newman ${ }^{\text {af }}$, A. Nisati ${ }^{\text {am }}$, T. Novak ${ }^{\text {ae }}$, H. Nowak ${ }^{\text {av }}$, R. Ofierzynski ${ }^{\text {aw }}$, G. Organtini ${ }^{\text {am }}$, I. Pal $^{\text {at }}$, C. Palomares ${ }^{\mathrm{y}}$, P. Paolucci ${ }^{\text {ac }}$, R. Paramatti ${ }^{\text {am }}$, G. Passaleva ${ }^{\text {q }}$, S. Patricelli ${ }^{\text {ac }}$, T. Paul ${ }^{\text {j }}$, M. Pauluzzi ${ }^{\text {ag }}$, C. Paus ${ }^{\mathrm{m}}$, F. Pauss ${ }^{\text {aw }}$, M. Pedace ${ }^{\text {am }}$, S. Pensotti ${ }^{\text {aa }}$, D. Perret-Gallix ${ }^{\mathrm{d}}$, D. Piccolo ${ }^{\text {ac }}$, F. Pierella ${ }^{\text {h }}$, M. Pioppi ${ }^{\text {ag }}$, P.A. Piroué ${ }^{\mathrm{ak}}$, E. Pistolesi ${ }^{\text {aa }}$, V. Plyaskin ${ }^{\text {ab }}$, M. Pohl ${ }^{\mathrm{t}}$, V. Pojidaev ${ }^{\mathrm{q}}$, J. Pothier ${ }^{\mathrm{r}}$, D. Prokofiev ${ }^{\text {ah }}$, G. Rahal-Callot ${ }^{\text {aw }}$, M.A. Rahaman ${ }^{\mathrm{i}}$, P. Raics ${ }^{\circ}$, N. Raja ${ }^{i}$, R. Ramelli ${ }^{\text {aw }}$, P.G. Rancoita ${ }^{\text {aa }}$, R. Ranieri $^{\mathrm{q}}$, A. Raspereza ${ }^{\text {av }}$, P. Razis ${ }^{\text {ad }}$, D. Ren ${ }^{\text {aw }}$, M. Rescigno ${ }^{\text {am }}$, S. Reucroft ${ }^{j}$, S. Riemann ${ }^{\text {av }}$, K. Riles ${ }^{\text {c }}$, B.P. Roe ${ }^{\text {c }}$, L. Romero ${ }^{y}$, A. Rosca ${ }^{\text {av }}$, C. Rosemann ${ }^{\text {a }}$, C. Rosenbleck ${ }^{\text {a }}$, S. Rosier-Lees ${ }^{\text {d }}$, S. Roth ${ }^{\text {a }}$, J.A. Rubio ${ }^{\text {r }}$, G. Ruggiero ${ }^{\text {q }}$, H. Rykaczewski ${ }^{\text {aw }}$, A. Sakharov ${ }^{\text {aw }}$, S. Saremi ${ }^{\text {f }}$, S. Sarkar ${ }^{\text {am }}$,

J. Salicio ${ }^{\mathrm{r}}$, E. Sanchez ${ }^{\mathrm{y}}$, C. Schäfer ${ }^{\mathrm{r}}$, V. Schegelsky ${ }^{\text {ah }}$, S. Schmidt-Kaerst ${ }^{\mathrm{a}}$, H. Schopper ${ }^{u}$, D.J. Schotanus ${ }^{\text {ae }}$, C. Sciacca ${ }^{\text {ac }}$, L. Servoli ${ }^{\text {ag }}$, S. Shevchenko ${ }^{\text {af }}$, N. Shivarov ${ }^{\text {ap }}$, V. Shoutko ${ }^{\mathrm{m}}$, E. Shumilov ${ }^{\mathrm{ab}}$, A. Shvorob ${ }^{\text {af }}$, D. Son ${ }^{\mathrm{aq}}$, C. Souga ${ }^{\mathrm{x}}$, P. Spillantini ${ }^{\mathrm{q}}$, M. Steuer ${ }^{\mathrm{m}}$, D.P. Stickland ${ }^{\text {ak }}$, B. Stoyanov ${ }^{\text {ap }}$, A. Straessner ${ }^{\mathrm{t}}$, K. Sudhakar ${ }^{i}$, G. Sultanov ${ }^{\text {ap }}$, L.Z. Sun ${ }^{v}$, S. Sushkov ${ }^{\text {a }}$, H. Suter ${ }^{\text {aw }}$, J.D. Swain ${ }^{j}$, Z. Szillasi ${ }^{z, 2}$, X.W. Tang ${ }^{g}$, P. Tarjan ${ }^{\circ}$, L. Tauscher ${ }^{\mathrm{e}}$, L. Taylor ${ }^{\mathrm{j}}$, B. Tellili ${ }^{\mathrm{x}}$, D. Teyssier ${ }^{\mathrm{x}}$, C. Timmermans ${ }^{\mathrm{ae}}$, Samuel C.C. Ting ${ }^{\mathrm{m}}$, S.M. Ting ${ }^{\mathrm{m}}$, S.C. Tonwar ${ }^{\mathrm{i}}$, J. Tóth ${ }^{1}$, C. Tully ${ }^{\text {ak }}$, K.L. Tung ${ }^{\mathrm{g}}$, J. Ulbricht ${ }^{\text {aw }}$, E. Valente ${ }^{\mathrm{am}}$, R.T. Van de Walle ${ }^{\text {ae }}$, R. Vasquez ${ }^{\text {at }}$, V. Veszpremi ${ }^{\text {z }}$, G. Vesztergombi ${ }^{1}$, I. Vetlitsky ${ }^{\text {ab }}$, G. Viertel ${ }^{\text {aw }}$, S. Villa ${ }^{\text {al }}$, M. Vivargent ${ }^{\mathrm{d}}$, S. Vlachos ${ }^{\mathrm{e}}$, I. Vodopianov ${ }^{\mathrm{z}}$, H. Vogel ${ }^{\text {ai }}$, H. Vogt ${ }^{\text {av }}$, I. Vorobiev ${ }^{\text {ai,ab }}$, A.A. Vorobyov ${ }^{\text {ah }}$, M. Wadhwa ${ }^{\mathrm{e}}$, Q. Wang ${ }^{\text {ae }}$, X.L. Wang ${ }^{\mathrm{v}}$, Z.M. Wang ${ }^{\mathrm{v}}$, M. Weber ${ }^{\mathrm{r}}$, S. Wynhoff ${ }^{\text {ak }}$, L. Xia ${ }^{\text {af }}$, Z.Z. Xu ${ }^{v}$, J. Yamamoto ${ }^{c}$, B.Z. Yang ${ }^{v}$, C.G. Yang ${ }^{g}$, H.J. Yang ${ }^{c}$, M. Yang ${ }^{\mathrm{g}}$, S.C. Yeh ${ }^{\text {as }}$, An. Zalite ${ }^{\text {ah }}$, Yu. Zalite ${ }^{\text {ah }}$, Z.P. Zhang ${ }^{\mathrm{v}}$, J. Zhao ${ }^{\mathrm{v}}$, G.Y. Zhu ${ }^{\mathrm{g}}$, R.Y. Zhu ${ }^{\text {af }}$, H.L. Zhuang ${ }^{\mathrm{g}}$, A. Zichichi ${ }^{\text {h,r,s }}$, B. Zimmermann ${ }^{\text {aw }}$, M. Zöller ${ }^{\text {a }}$

${ }^{a}$ III. Physikalisches Institut, RWTH, D-52056 Aachen, Germany ${ }^{3}$

${ }^{\mathrm{b}}$ National Institute for High Energy Physics, NIKHEF, and University of Amsterdam, NL-1009 DB Amsterdam, The Netherlands c University of Michigan, Ann Arbor, MI 48109, USA

d Laboratoire d'Annecy-le-Vieux de Physique des Particules, LAPP, IN2P3-CNRS, BP 110, F-74941 Annecy-le-Vieux cedex, France ${ }^{\mathrm{e}}$ Institute of Physics, University of Basel, CH-4056 Basel, Switzerland 


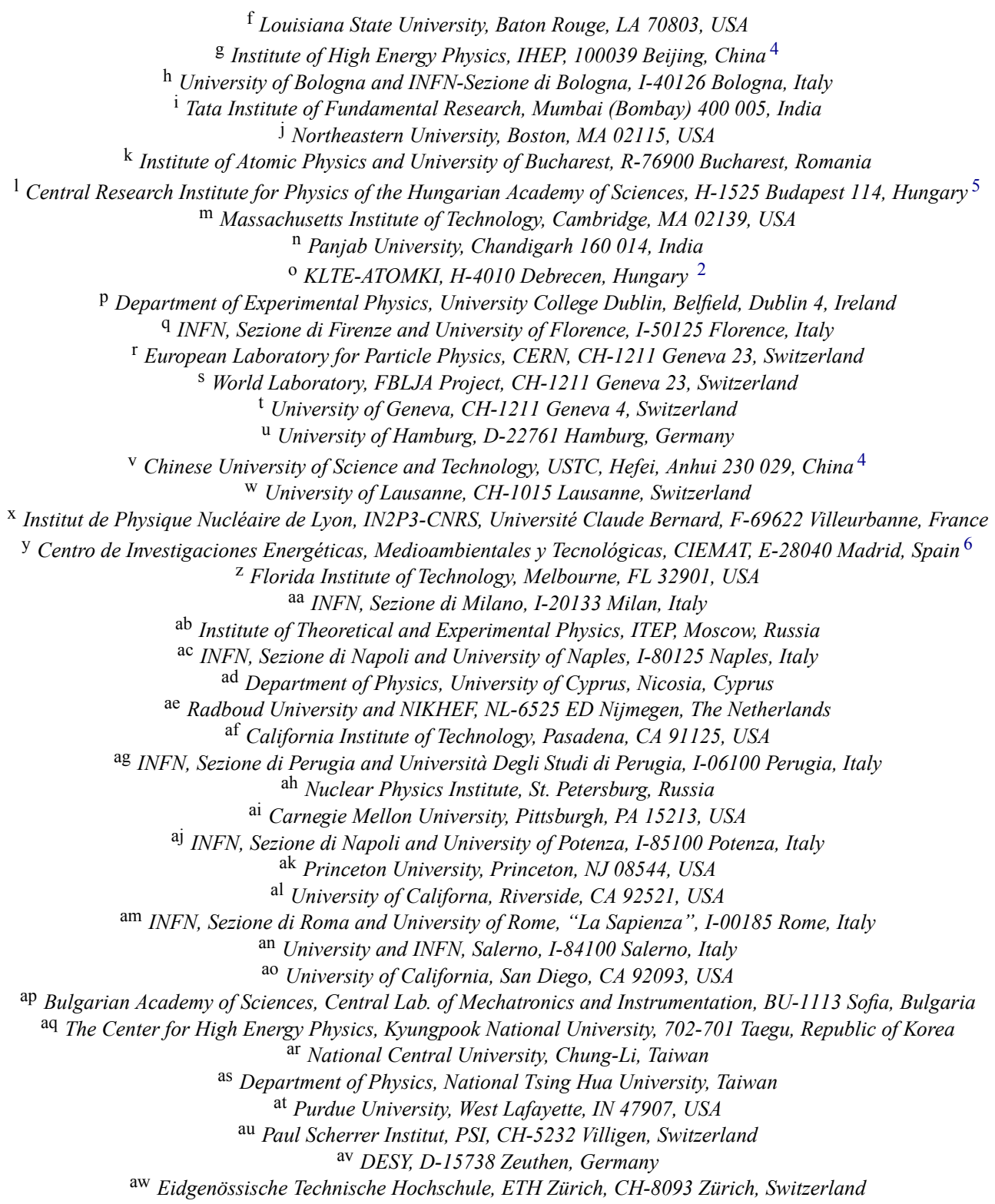

Received 4 March 2005; accepted 6 April 2005

Available online 4 May 2005

Editor: L. Rolandi

\begin{abstract}
Compton scattering of quasi-real virtual photons, $\gamma \mathrm{e}^{ \pm} \rightarrow \gamma \mathrm{e}^{ \pm}$, is studied with $0.6 \mathrm{fb}^{-1}$ of data collected by the L3 detector at the LEP $\mathrm{e}^{+} \mathrm{e}^{-}$collider at centre-of-mass energies $\sqrt{s}=189-209 \mathrm{GeV}$. About 4500 events produced by the interaction of virtual photons emitted by $\mathrm{e}^{ \pm}$of one beam with $\mathrm{e}^{\mp}$ of the opposite beam are collected for effective centre-of-mass energies of
\end{abstract}


the photon-electron and photon-positron systems in the range from $\sqrt{s^{\prime}}=35 \mathrm{GeV}$ up to $\sqrt{s^{\prime}}=175 \mathrm{GeV}$, the highest energy at which Compton scattering was ever probed. The cross sections of the $\gamma \mathrm{e}^{ \pm} \rightarrow \gamma \mathrm{e}^{ \pm}$process as a function of $\sqrt{s^{\prime}}$ and of the rest-frame scattering angle are measured, combined with previous L3 measurements down to $\sqrt{s^{\prime}} \simeq 20 \mathrm{GeV}$, and found to agree with the QED expectations.

๑) 2005 Published by Elsevier B.V.

\section{Introduction}

The CERN LEP $\mathrm{e}^{+} \mathrm{e}^{-}$collider allowed high-energy tests of high-order QED through the detection of events with multiple hard-photon production [1] and the study of lepton pairs produced by two-photon interactions [2].

A unique test of QED at $\mathrm{e}^{+} \mathrm{e}^{-}$colliders is the study of Compton scattering, $\gamma \mathrm{e}^{ \pm} \rightarrow \gamma \mathrm{e}^{ \pm}$. In this process, quasi-real virtual photons ${ }^{7}$ emitted by one of the incoming beams interact with the electrons ${ }^{8}$ of the other beam. This process, $\mathrm{e}^{+} \mathrm{e}^{-} \rightarrow \mathrm{e}^{+} \mathrm{e}^{-} \gamma$, is sketched in Fig. 1. The electron which radiates the quasi-real photon is scattered at a very small angle and escapes detection along the beam pipe. The signature of such a process is a photon and an electron in the detector, with a large amount of missing momentum directed along the beam line. The $\gamma \mathrm{e}^{ \pm} \rightarrow \gamma \mathrm{e}^{ \pm}$process is characterised by the effective centre-of-mass energy of the photon-electron collision, $\sqrt{s^{\prime}}$, and by the scattering angle of the electron in the photon-electron centre-ofmass system, $\theta^{*}$, shown in Fig. 1 . At the lowest order,

\footnotetext{
${ }^{1}$ Also supported by CONICET and Universidad Nacional de La Plata, CC 67, 1900 La Plata, Argentina.

2 Also supported by the Hungarian OTKA fund under contract number T026178.

3 Supported by the German Bundesministerium für Bildung, Wissenschaft, Forschung und Technologie.

${ }^{4}$ Supported by the National Natural Science Foundation of China.

5 Supported by the Hungarian OTKA fund under contract numbers T019181, F023259 and T037350.

6 Supported also by the Comisión Interministerial de Ciencia y Tecnología.

7 A "quasi-real" virtual photon is one whose virtuality, $Q^{2}=$ $-m_{\gamma}^{2}$, is much smaller than all relevant scales of the process and is therefore kinematically equivalent to a real photon with $Q^{2}=0$.

${ }^{8}$ In this Letter, the term "electron" is in general used to refer to both electrons and positrons, unless specified otherwise.
}
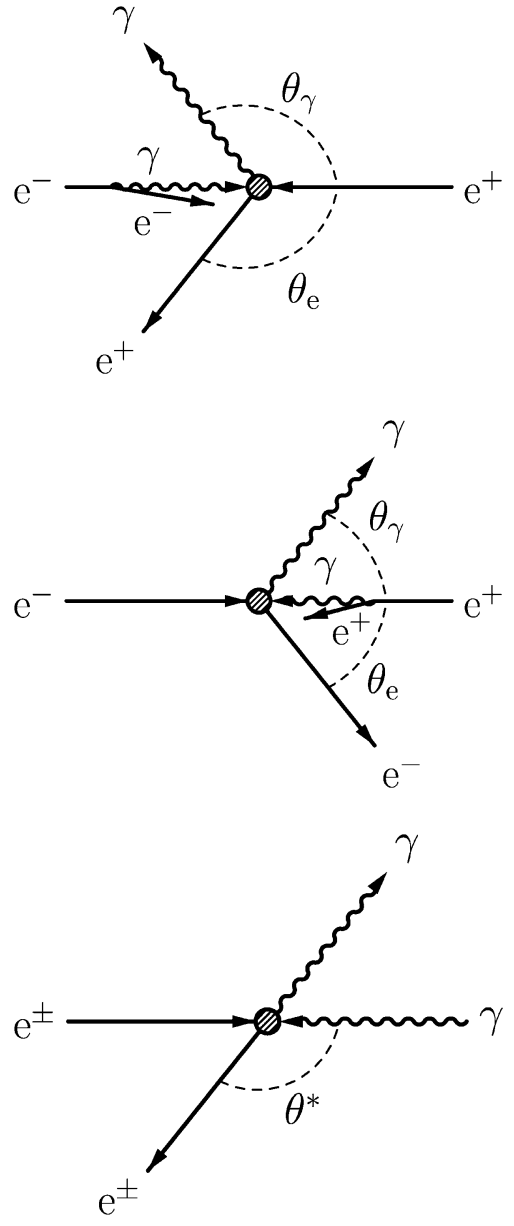

Fig. 1. Schematic view of quasi-real Compton scattering in $\mathrm{e}^{+} \mathrm{e}^{-}$ collisions in the laboratory system, higher plots, and in the $\gamma \mathrm{e}^{ \pm}$ centre-of-mass frame, lowest plot.

the differential cross section as a function of $\cos \theta^{*}$ is [3]:

$$
\frac{\mathrm{d} \sigma}{\mathrm{d} \cos \theta^{*}}=\frac{\alpha^{2} \pi}{s^{\prime}}\left(\frac{1+\cos \theta^{*}}{2}+\frac{2}{1+\cos \theta^{*}}\right),
$$

where $\alpha$ is the fine-structure constant. In the angular range $\left|\cos \theta^{*}\right|<0.8$, this corresponds to a cross sec- 
Table 1

Integrated luminosities, $\mathcal{L}$, and numbers of events selected in data, $N_{\text {data }}$, and Monte Carlo, $N_{\mathrm{MC}}$, at each $\mathrm{e}^{+} \mathrm{e}^{-}$centre-of-mass energy

\begin{tabular}{llllllll}
\hline$\sqrt{s}(\mathrm{GeV})$ & 188.6 & 191.6 & 195.6 & 199.5 & 201.7 & $202.5-205.5$ & $205.5-209.2$ \\
$\mathcal{L}\left(\mathrm{pb}^{-1}\right)$ & 176.8 & 28.9 & 82.9 & 67.8 & 36.2 & 75.6 & 137.7 \\
$N_{\text {data }}$ & 1409 & 221 & 612 & 460 & 266 & 545 & 974 \\
$N_{\text {MC }}$ & 1336 & 231 & 659 & 521 & 284 & 520 & 982 \\
\hline
\end{tabular}

tion of about $200 \mathrm{pb}$ at $\sqrt{s^{\prime}}=40 \mathrm{GeV}$ and of about $20 \mathrm{pb}$ at $\sqrt{s^{\prime}}=130 \mathrm{GeV}$.

Quasi-real Compton scattering in $\mathrm{e}^{+} \mathrm{e}^{-}$colliders was first discussed more than three decades ago [4] and observed at the $900 \mathrm{MeV}$ ACO storage ring in Orsay [5]. The L3 Collaboration studied this process at $\mathrm{e}^{+} \mathrm{e}^{-}$centre-of-mass energies $\sqrt{s}=91-183 \mathrm{GeV}$, covering with high statistics the effective centre-ofmass energy range $\sqrt{s^{\prime}}=20-100 \mathrm{GeV}$ [6]. This Letter presents the extension of this analysis to the highluminosity and high-energy data sample collected at LEP at $\sqrt{s}=189-209 \mathrm{GeV}$ with the L3 detector [79]. This data sample comprises $0.6 \mathrm{fb}^{-1}$ of integrated luminosity, as detailed in Table 1 . It extends the accessible $\sqrt{s^{\prime}}$ range to about $175 \mathrm{GeV}$, allowing to probe Compton scattering at energies never attained before.

\section{Event simulation}

The TEEGG Monte Carlo [10] is used to simulate the $\mathrm{e}^{+} \mathrm{e}^{-} \rightarrow \mathrm{e}^{+} \mathrm{e}^{-} \gamma$ process with one electron scattered in the angular range $|\cos \theta|>0.996$ and both the other electron and the photon in the angular range $|\cos \theta|<0.985$. The $\sqrt{s^{\prime}}$ spectrum for the $\gamma \mathrm{e}^{ \pm} \rightarrow$ $\gamma \mathrm{e}^{ \pm}$process generated by TEEGG was compared [11] with the QED expectations obtained by convolving the QED cross section with the virtual-photon flux modelled with the equivalent-photon approach [12]. The two spectra agree within the expected statistical precision of this measurement.

The following Monte Carlo programs are used to model the background processes: BHWIDE [13] for Bhabha scattering, $\mathrm{e}^{+} \mathrm{e}^{-} \rightarrow \mathrm{e}^{+} \mathrm{e}^{-}$, KK2f [14] for tau pair-production, $\mathrm{e}^{+} \mathrm{e}^{-} \rightarrow \tau^{+} \tau^{-}$, GGG [15] for multiphoton production, $\mathrm{e}^{+} \mathrm{e}^{-} \rightarrow \gamma \gamma(\gamma)$, and DIAG36 [16] for electron pair-production in two-photon collisions, $\mathrm{e}^{+} \mathrm{e}^{-} \rightarrow \mathrm{e}^{+} \mathrm{e}^{-} \mathrm{e}^{+} \mathrm{e}^{-}$.

Large samples of Monte Carlo events are generated at each $\mathrm{e}^{+} \mathrm{e}^{-}$centre-of-mass energy. The number of simulated signal events corresponds to at least thirty times the number of expected data events. For background processes, this factor varies from six times for Bhabha scattering, up to more than one hundred times for the $\mathrm{e}^{+} \mathrm{e}^{-} \rightarrow \tau^{+} \tau^{-}$process.

The L3 detector response is simulated using the GEANT program [17], which takes into account the effects of energy loss, multiple scattering and showering in the detector. Time-dependent efficiencies of the different subdetectors, as monitored during the datataking period, are taken into account in the simulation procedure. The simulated events are reconstructed with the same program used for the data.

\section{Reconstruction of event kinematics}

A crucial part of the measurement is the determination of $\sqrt{s^{\prime}}$ and $\cos \theta^{*}$. These are inferred with high precision by imposing the constraints that there are only three particles in the final state and one of the electrons is directed along the beam line. The polar angles of the observed electron and photon, $\theta_{\mathrm{e}}$ and $\theta_{\gamma}$, defined in Fig. 1, are used to calculate the missing energy, $E_{\text {miss }}$, as:

$E_{\text {miss }}=\sqrt{s} \frac{\left|\sin \left(\theta_{\mathrm{e}}+\theta_{\gamma}\right)\right|}{\sin \theta_{\mathrm{e}}+\sin \theta_{\gamma}+\left|\sin \left(\theta_{\mathrm{e}}+\theta_{\gamma}\right)\right|}$.

The square of the effective centre-of-mass energy of the $\gamma \mathrm{e}^{ \pm} \rightarrow \gamma \mathrm{e}^{ \pm}$process is then:

$s^{\prime}=s\left(1-2 \frac{E_{\mathrm{miss}}}{\sqrt{s}}\right)$.

Monte Carlo studies show that the resolution on $\sqrt{s^{\prime}}$ is better than $500 \mathrm{MeV}$. This improves by $30 \%$ the resolution obtained if only the energies of the measured particles are used. The resolution on the measured energies of the electron, $E_{\mathrm{e}}$, and of the photon $E_{\gamma}$ is also improved by recalculating these quantities with 
the kinematic constraints:

$$
\begin{aligned}
& E_{\mathrm{e}}^{\text {angle }}=\sqrt{s} \frac{\sin \theta_{\gamma}}{\sin \theta_{\mathrm{e}}+\sin \theta_{\gamma}+\left|\sin \left(\theta_{\mathrm{e}}+\theta_{\gamma}\right)\right|}, \\
& E_{\gamma}^{\text {angle }}=\sqrt{s} \frac{\sin \theta_{\mathrm{e}}}{\sin \theta_{\mathrm{e}}+\sin \theta_{\gamma}+\left|\sin \left(\theta_{\mathrm{e}}+\theta_{\gamma}\right)\right|} .
\end{aligned}
$$

Both $E_{\mathrm{e}}^{\text {angle }}$ and $E_{\gamma}^{\text {angle }}$ have a resolution of about 200 $\mathrm{MeV}$. The cosine of the scattering angle of the electron in the electron-photon centre-of-mass system is:

$$
\cos \theta^{*}=\frac{\sin \left(\theta_{\gamma}-\theta_{\mathrm{e}}\right)}{\sin \theta_{\gamma}+\sin \theta_{\mathrm{e}}} \text {. }
$$

The resolution on $\cos \theta^{*}$ in Monte Carlo events is found to be better than 0.005 .

\section{Event selection}

Events from Compton scattering must have one track in the central tracker and two clusters in the BGO electromagnetic calorimeter in the fiducial volume $|\cos \theta|<0.96$. The clusters must have energies of at least $5 \mathrm{GeV}$ and the lateral profile of their showers must match that expected for electromagnetic showers. One of these clusters must be associated to the track, which should be reconstructed from at least $80 \%$ of the hits along its sensitive track-length. This cluster is identified as the electron.

The low polar-angle regions of the L3 detector are instrumented with two calorimeters. The first is composed of BGO crystals and is used to detect Bhabha scattering in order to measure the luminosity [8]. It covers the angular region $1.4^{\circ}<\theta<3.9^{\circ}$. The second is built from lead and plastic scintillators and extends this angular coverage up to $9^{\circ}$ [9]. The sum of the energies deposited in these two calorimeters, $E_{\text {forward }}$, is required to be less than $50 \mathrm{GeV}$, as shown in Fig. 2a. This cut ensures that no electron is detected above $1.4^{\circ}$ and therefore only the scattering of quasi-real photons is considered.

After these preselection requirements, about 36500 events are observed in data and 32500 are expected from Monte Carlo processes, of which $18 \%$ is due to signal and $82 \%$ to background. The main source of background is Bhabha scattering with a highenergy initial-state-radiation (ISR) photon emitted in the beam pipe, which gives the missing-energy signature, and an electron which mimics a photon. Electrons are misidentified as photons more frequently in data than in Monte Carlo, which results in the excess of observed events over the Monte Carlo prediction. An additional contribution to this background comes from tau pair-production where both taus decay into electrons, one of which is identified as a photon, and the four neutrinos are responsible for the missing-energy signature. A lesser source of background is the production of events with three photons, where one of the photons escapes detection along the beam line and another, through photon conversion, is mistaken for an electron. Several additional criteria are devised to cope with these background sources.

Bhabha scattering and multi-photon production favour events with electromagnetic clusters which are back-to-back in space. These background sources are strongly reduced by a cut on the angle between the two clusters, $\zeta<2.8$ rad, as illustrated in Fig. $2 \mathrm{~b}$.

Events from tau pair-production are suppressed by requiring $E_{\mathrm{e}} / E_{\mathrm{e}}^{\text {angle }}>0.7$ if $E_{\mathrm{e}}>E_{\gamma}$ or $E_{\gamma} / E_{\gamma}^{\text {angle }}>$ 0.7 otherwise, as shown in Fig. 2c. This cut enforces the three-particle hypothesis and rejects events where the missing momentum is not directed along the beam axis.

The analysis is restricted to $\sqrt{s^{\prime}}>35 \mathrm{GeV}$, as displayed in Fig. 2d, in order to exclude the region L3 measured with high statistics [6] and concentrate on the high-energy data.

The background from Bhabha scattering is mainly concentrated in the forward scattering region, $\cos \theta^{*}>$ 0.8 , as presented in Fig. 2e. This region is removed from the analysis. The backward-scattering region, $\cos \theta^{*}<-0.8$, is also removed in order to reduce the systematic uncertainty from a wrong assignment of the electron charge.

The contribution from Bhabha scattering to the selected events is further inspected. In the forward scattering region, $0.4<\cos \theta^{*}<0.8$, the background electrons are mostly emitted in the central regions of the detector, as shown in Fig. 2f. This follows from the emission of a hard ISR photon. This background is further reduced by requiring the electron to satisfy $\left|\cos \theta_{\mathrm{e}}\right|>0.6$ if $0.4<\cos \theta^{*}<0.8$.

After these cuts, 4487 events are selected in data and 4534 are expected from Monte Carlo simulations, as detailed in Table 1 [18]. Background processes are estimated to contribute to about $3.5 \%$ of this sample. Two thirds of the background is due to Bhabha scatter- 

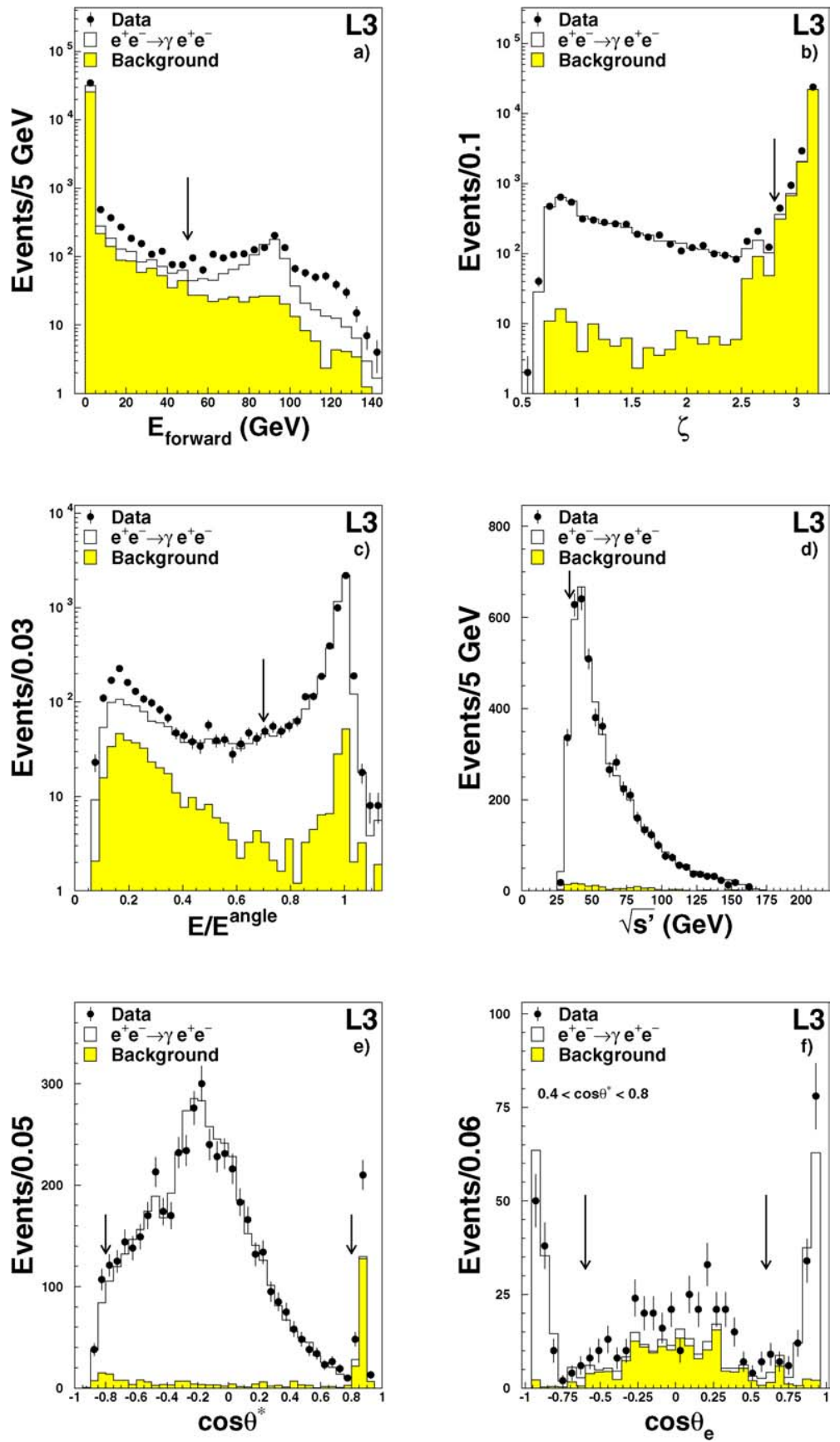

Fig. 2. Distributions of some selection variables for data and Monte Carlo predictions. (a) Energy in the forward calorimeters, $E_{\text {forward }}$; (b) angle between the electron and the photon, $\zeta$; (c) ratio of the energies of the most energetic particle as measured in the calorimeter and as obtained from the angular constraints, $E / E^{\text {angle }}$; (d) effective centre-of-mass energy, $\sqrt{s^{\prime}} ;$ (e) cosine of the electron scattering angle in the $\gamma \mathrm{e}^{ \pm}$rest frame, $\cos \theta^{*}$; (f) cosine of the polar angle of the electron, $\cos \theta_{\mathrm{e}}$, for $0.4<\cos \theta^{*}<0.8$. The arrows indicate the position of the cuts. In (a) all other pre-selection cuts are applied. In (b) all cuts are applied but the one on $\cos \theta^{*}$. In (c)-(f) cuts on all other variables are applied. 

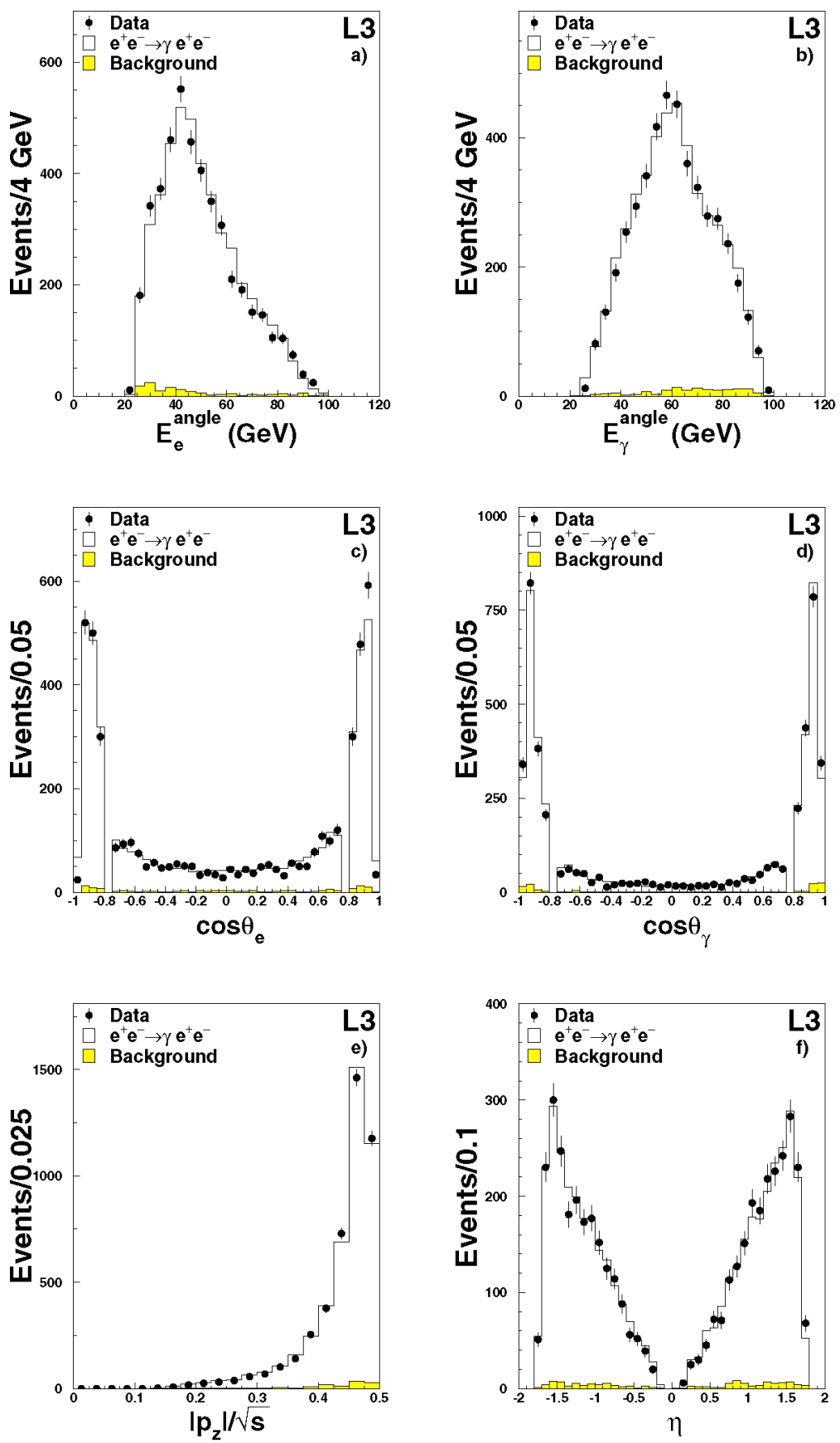

Fig. 3. Distributions of variables for events selected in data and in the signal and background Monte Carlo samples. Energy of (a) the electron, $E_{\mathrm{e}}^{\text {angle }}$, and (b) the photon, $E_{\gamma}^{\text {angle }}$; cosine of the polar angle of (c) the electron, $\theta_{\mathrm{e}}$, and (d) the photon, $\theta_{\gamma} ;$ (e) normalised sum of the electron and photon longitudinal momenta, $\left|p_{\mathbf{Z}}\right| / \sqrt{s}$; (f) rapidity of the event, $\eta$. 

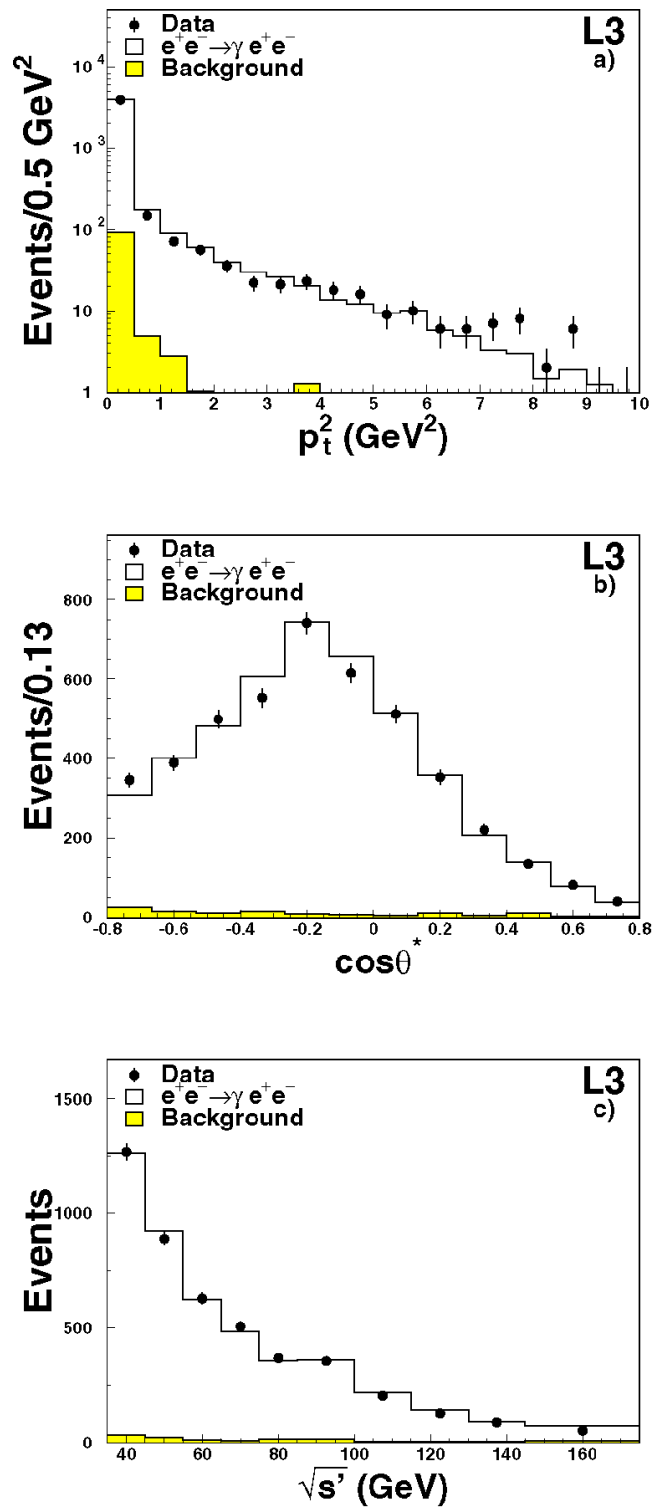

Fig. 4. Distributions of variables for events selected in data and in the signal and background Monte Carlo samples. (a) Square of the sum of the electron and photon transverse momenta, $p_{\mathrm{t}}^{2} ;(\mathrm{b})$ cosine of the electron scattering angle in the $\gamma \mathrm{e}^{ \pm}$rest frame, $\cos \theta^{*}$; (c) effective centre-of-mass energy, $\sqrt{s^{\prime}}$.

ing, one quarter to electron production in two photon collisions and the rest to multi-photon production and tau pair-production. The distributions of the energies and the angles of the electrons and photons of the selected events are shown in Figs. 3a-d. Fig. 3e presents the normalised sum of the momenta of the electron and
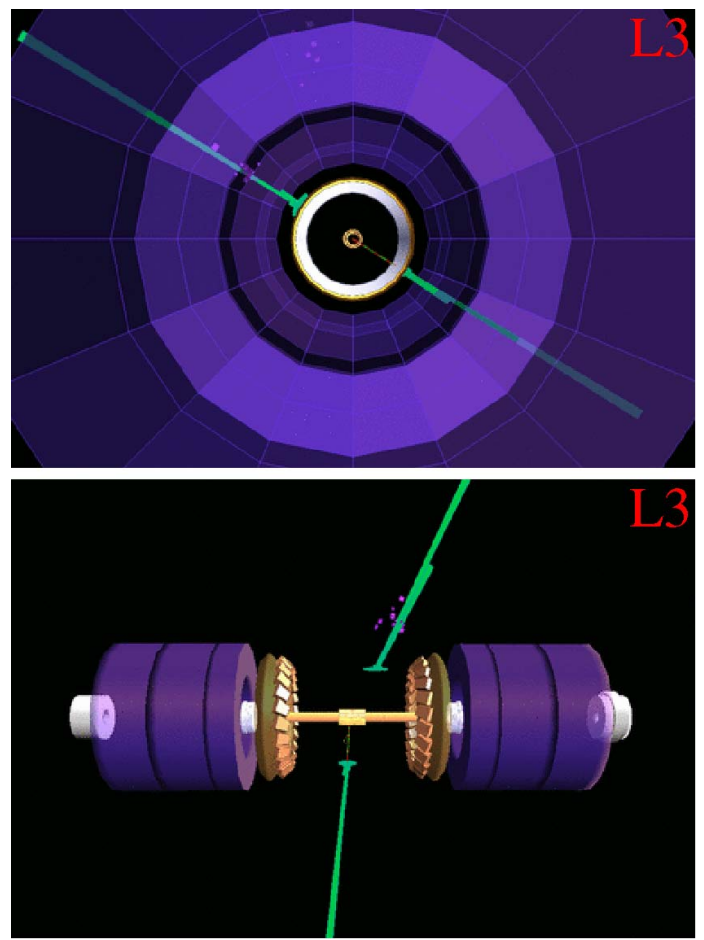

Fig. 5. Views of a high-energy candidate for quasi-real Compton scattering in the plane transverse to the beams, higher plot, and in a plane along the beams, lower plot. The towers represent the energy deposited in the electromagnetic calorimeter and the boxes are low-energy clusters in the hadron calorimeter. The track of the electron is clearly visible, while no track is associated to the other electromagnetic cluster, identified as the photon.

photon along the beam line, $\left|p_{\mathrm{z}}\right| / \sqrt{s}$. As expected, a large boost along the beam line is observed. Fig. $3 \mathrm{f}$ shows the rapidity of the selected events.

Monte Carlo studies indicate that the average value of $Q^{2}$ for the selected events is $0.48 \mathrm{GeV}^{2}$ and $90 \%$ of the photons satisfy $Q^{2}<2 \mathrm{GeV}^{2}$. This corresponds to a small average-virtuality $\left\langle Q^{2} / s^{\prime}\right\rangle \simeq 3 \times 10^{-3}$, which justifies the treatment of the virtual photons as quasireal ones. The kinematics of the process under investigation is such that $p_{\mathrm{t}}^{2} \simeq Q^{2}$, where $p_{\mathrm{t}}$ is the sum of the momenta of the final state electron and photon in the plane transverse to the beams. Fig. 4a shows the distribution of $p_{\mathrm{t}}^{2}$ for the selected events. An average value $\left\langle p_{\mathrm{t}}^{2}\right\rangle=0.3 \mathrm{GeV}^{2}$ is observed, with a root-mean-square of $1 \mathrm{GeV}^{2}$, which further validates the hypothesis of quasi-real photons.

The distribution of $\cos \theta^{*}$ for the selected events, shown in Fig. 4b, presents the characteristic backward- 
Table 2

Systematic uncertainties on the cross section as a function of $\sqrt{s^{\prime}}$ and of the differential cross section as a function of $\cos \theta^{*}$

\begin{tabular}{lll}
\hline & \multicolumn{2}{l}{ Systematic uncertainty on } \\
\cline { 2 - 3 } Source & $\sigma_{\gamma \mathrm{e}^{ \pm} \rightarrow \gamma \mathrm{e}^{ \pm}\left(\sqrt{s^{\prime}}\right)} \mathrm{d} \sigma_{\gamma \mathrm{e}^{ \pm} \rightarrow \gamma \mathrm{e}^{ \pm} / \mathrm{d} \cos \theta^{*}}$ \\
\hline Measurement of angles & $0.2 \%-3.8 \%$ & $0.1 \%-18.6 \%$ \\
Energy scale & $0.1 \%-0.9 \%$ & $0.1 \%-1.9 \%$ \\
Charge confusion & $0.1 \%-0.2 \%$ & $0.1 \%-19.8 \%$ \\
Background normalisation & $0.1 \%-0.2 \%$ & $0.1 \%-0.3 \%$ \\
Signal Monte Carlo statistics & $1.0 \%-3.7 \%$ & $1.3 \%-6.9 \%$ \\
Background Monte Carlo statistics & $0.3 \%-2.4 \%$ & $0.3 \%-4.2 \%$ \\
Total & $1.3 \%-5.5 \%$ & $1.5 \%-28.2 \%$ \\
\hline
\end{tabular}

The largest uncertainties correspond to the low-statistics high $\sqrt{s^{\prime}}$ regions and to the forward-scattering regions, where angular measurements and charge confusion effects are important.

Table 3

Number of events observed in each $\sqrt{s^{\prime}}$ bin with average $\left\langle\sqrt{s^{\prime}}\right\rangle, N_{\text {data }}$, together with the signal, $N_{\mathrm{MC}}^{\text {sign }}$, and background, $N_{\text {MC }}^{\text {back }}$, Monte Carlo predictions

\begin{tabular}{ccrrrrr}
\hline$\sqrt{s^{\prime}}(\mathrm{GeV})$ & $\left\langle\sqrt{s^{\prime}}\right\rangle(\mathrm{GeV})$ & $N_{\text {data }}$ & $N_{\mathrm{MC}}^{\text {sign }}$ & $N_{\mathrm{MC}}^{\text {back }}$ & $\sigma_{\gamma \mathrm{e}^{ \pm} \rightarrow \gamma \mathrm{e}^{ \pm}(\mathrm{pb})}$ & $\sigma_{\gamma \mathrm{e}^{ \pm} \rightarrow \gamma \mathrm{e}^{ \pm}}^{\mathrm{QED}}(\mathrm{pb})$ \\
\hline $35-45$ & 39.7 & 1269 & 1229.7 & 32.0 & $216.0 \pm 6.0 \pm 4.7$ & 214.7 \\
$45-55$ & 49.7 & 889 & 900.3 & 22.5 & $131.5 \pm 4.6 \pm 1.8$ & 136.7 \\
$55-65$ & 59.8 & 627 & 610.6 & 11.8 & $95.3 \pm 3.8 \pm 2.2$ & 94.6 \\
$65-75$ & 69.8 & 506 & 472.5 & 9.8 & $72.9 \pm 3.1 \pm 1.5$ & 69.4 \\
$75-85$ & 79.8 & 370 & 342.4 & 16.5 & $54.8 \pm 2.8 \pm 1.2$ & 53.1 \\
$85-100$ & 92.2 & 357 & 346.9 & 14.9 & $39.2 \pm 2.1 \pm 1.1$ & 39.8 \\
$100-115$ & 107.2 & 205 & 214.4 & 5.8 & $27.3 \pm 2.1 \pm 0.8$ & 29.4 \\
$115-130$ & 122.3 & 125 & 138.2 & 2.6 & $20.0 \pm 2.0 \pm 0.7$ & 22.6 \\
$130-145$ & 137.3 & 87 & 86.6 & 3.3 & $17.3 \pm 1.9 \pm 0.9$ & 17.9 \\
$145-175$ & 159.3 & 52 & 66.6 & 6.5 & $9.1 \pm 1.8 \pm 0.7$ & 13.3 \\
\hline
\end{tabular}

The measured cross sections, $\sigma_{\gamma \mathrm{e}^{ \pm} \rightarrow \gamma \mathrm{e}^{ \pm}}$, are given with their statistical and systematic uncertainties, respectively, together with the QED predictions, $\sigma_{\gamma \mathrm{e}^{ \pm} \rightarrow \gamma \mathrm{e}^{ \pm}}^{\mathrm{QED}}$. The data sample at $\sqrt{s}=188.6-209.2 \mathrm{GeV}$ is considered, and the cosine of the electron rest-frame scattering angle is limited to the range $\left|\cos \theta^{*}\right|<0.8$.

scattering behaviour of Compton scattering. Fig. 4c displays the observed and expected distributions of $\sqrt{s^{\prime}}$. The average value of $\sqrt{s^{\prime}}$ is $64 \mathrm{GeV}$. Ten events are observed in data for $\sqrt{s^{\prime}}>163 \mathrm{GeV}$, up to $\sqrt{s^{\prime}}=$ $175 \mathrm{GeV}$, the largest energies at which Compton scattering was ever observed. Monte Carlo simulations predict $9 \pm 1$ events in this region, with a background of $7 \%$. The uncertainty is due to the limited statistics of the generated Monte Carlo sample. Fig. 4 presents one of these high-energy events.

\section{Systematic uncertainties}

Several sources of systematic uncertainty are considered and their impact on the measurement of the cross section as a function of $\sqrt{s^{\prime}}$ and of the differen- tial cross section as a function of $\cos \theta^{*}$ are listed in Table 2.

The measurement of the photon and electron angles is crucial to the determination of the event kinematics. These variables are smeared by $\pm 1 \%$ to account for possible uncertainties in detector alignment, timedependent changes in resolution or discrepancies between the data and the Monte Carlo simulations. The effects of these changes are larger for events with large values of $\sqrt{s^{\prime}}$ or $\cos \theta^{*}$. In addition, the energy scale of the electromagnetic calorimeter is varied within its uncertainty of $\pm 1 \%$. As the energies are mainly inferred from the angular measurements, this change has a small impact on the cross sections.

The amount of charge confusion in the tracker is measured with control data-samples and is well re- 
Table 4

Number of events observed in each $\cos \theta^{*}$ bin with average $\left\langle\cos \theta^{*}\right\rangle, N_{\text {data }}$, together with the signal, $N_{\mathrm{MC}}^{\text {sign }}$, and background, $N_{\text {MC }}^{\text {back }}$, Monte Carlo predictions

\begin{tabular}{|c|c|c|c|c|c|c|}
\hline $\cos \theta^{*}$ & $\left\langle\cos \theta^{*}\right\rangle$ & $N_{\text {data }}$ & $N_{\mathrm{MC}}^{\mathrm{sign}}$ & $N_{\mathrm{MC}}^{\text {back }}$ & 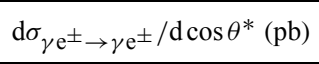 & $\mathrm{d} \sigma_{\gamma \mathrm{e}^{ \pm} \rightarrow \gamma \mathrm{e}^{ \pm}}^{\mathrm{QED}} / \mathrm{d} \cos \theta^{*}(\mathrm{pb})$ \\
\hline$-0.80--0.67$ & -0.74 & 346 & 283.3 & 25.8 & $133.8 \pm 6.4 \pm 9.6 \times 10^{2}$ & $118.3 \times 10^{2}$ \\
\hline$-0.67--0.53$ & -0.60 & 389 & 385.2 & 15.7 & $77.2 \pm 4.0 \pm 2.6 \times 10^{2}$ & $79.6 \times 10^{2}$ \\
\hline$-0.53--0.40$ & -0.47 & 499 & 469.9 & 11.2 & $63.6 \pm 2.7 \pm 2.5 \times 10^{2}$ & $61.2 \times 10^{2}$ \\
\hline$-0.40--0.27$ & -0.34 & 553 & 593.5 & 14.5 & $46.0 \pm 2.1 \pm 1.5 \times 10^{2}$ & $50.7 \times 10^{2}$ \\
\hline$-0.27--0.13$ & -0.20 & 742 & 733.8 & 10.1 & $43.1 \pm 1.6 \pm 0.7 \times 10^{2}$ & $44.1 \times 10^{2}$ \\
\hline$-0.13-0.0$ & -0.07 & 616 & 650.2 & 7.9 & $37.0 \pm 1.6 \pm 0.6 \times 10^{2}$ & $39.6 \times 10^{2}$ \\
\hline $0.0-0.13$ & 0.06 & 512 & 509.2 & 5.8 & $36.4 \pm 1.6 \pm 0.6 \times 10^{2}$ & $36.6 \times 10^{2}$ \\
\hline $0.13-0.27$ & 0.20 & 353 & 347.6 & 10.9 & $33.4 \pm 1.8 \pm 0.8 \times 10^{2}$ & $34.4 \times 10^{2}$ \\
\hline $0.27-0.40$ & 0.33 & 221 & 202.4 & 5.1 & $35.0 \pm 2.2 \pm 2.2 \times 10^{2}$ & $32.9 \times 10^{2}$ \\
\hline $0.40-0.53$ & 0.46 & 135 & 126.7 & 11.9 & $30.1 \pm 2.7 \pm 3.1 \times 10^{2}$ & $31.8 \times 10^{2}$ \\
\hline $0.53-0.67$ & 0.60 & 81 & 73.5 & 2.8 & $33.1 \pm 3.4 \pm 5.9 \times 10^{2}$ & $31.1 \times 10^{2}$ \\
\hline $0.67-0.80$ & 0.73 & 40 & 33.1 & 4.1 & $33.3 \pm 4.8 \pm 8.7 \times 10^{2}$ & $30.7 \times 10^{2}$ \\
\hline
\end{tabular}

The measured differential cross sections, $\mathrm{d} \sigma_{\gamma \mathrm{e}^{ \pm}} \rightarrow \gamma \mathrm{e}^{ \pm} / \mathrm{d} \cos \theta^{*}$, are given with their statistical and systematic uncertainties, respectively, together with the QED predictions, $\mathrm{d} \sigma_{\gamma \mathrm{e}^{ \pm} \rightarrow \gamma \mathrm{e}^{ \pm}}^{\mathrm{QED}} / \mathrm{d} \cos \theta^{*}$. The data sample at $\sqrt{s}=188.6-209.2 \mathrm{GeV}$ is considered, corresponding to an effective centre-of-mass energy range $\sqrt{s^{\prime}}=35-175 \mathrm{GeV}$.

produced in the Monte Carlo simulations. However, uncertainties in this simulation are a potential source of systematics. This is assessed by injecting in the simulations an additional amount of charge confusion. A small variation in the cross section as a function of $\sqrt{s^{\prime}}$ is observed, whereas a larger uncertainty affects the determination of the differential cross section for large values of $\cos \theta^{*}$.

Uncertainties of the background normalisation are also propagated to the final results. All background levels are varied by $\pm 2 \%$ with the exception of electron production in two-photon collisions, varied by $\pm 10 \%$.

Finally, the effects of the limited amount of signal and background Monte Carlo statistics are treated as additional systematic uncertainties.

\section{Results}

The phase space covered by this analysis, $35 \mathrm{GeV}<$ $\sqrt{s^{\prime}}<175 \mathrm{GeV}$ and $-0.8<\cos \theta^{*}<0.8$, is divided in ten intervals in $\sqrt{s^{\prime}}$ and twelve intervals in $\cos \theta^{*}$. The limits of these intervals are detailed in Tables 3 and 4 , together with their average values ${ }^{9}$ and the numbers of

\footnotetext{
9 The average values are calculated as suggested in Ref. [19].
}

\section{Table 5}

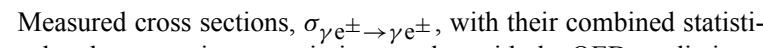
cal and systematic uncertainties together with the QED predictions, $\sigma$ QED

\begin{tabular}{|c|c|c|c|}
\hline$\sqrt{s^{\prime}}(\mathrm{GeV})$ & $\left\langle\sqrt{s^{\prime}}\right\rangle(\mathrm{GeV})$ & $\sigma_{\gamma \mathrm{e}^{ \pm} \rightarrow \gamma \mathrm{e}^{ \pm}}$ & $\begin{array}{l}\sigma^{\text {QED }} \\
\gamma \mathrm{e}^{ \pm} \rightarrow \gamma \mathrm{e}^{ \pm}\end{array}$ \\
\hline $0-25$ & 21.0 & $771.2 \pm 21.6$ & 764.8 \\
\hline $25-35$ & 29.8 & $370.6 \pm 11.3$ & 381.1 \\
\hline $35-45$ & 39.7 & $213.2 \pm 5.4$ & 214.7 \\
\hline $45-55$ & 49.7 & $128.7 \pm 3.9$ & 136.7 \\
\hline $55-65$ & 59.8 & $95.0 \pm 3.5$ & 94.6 \\
\hline $65-75$ & 69.8 & $70.6 \pm 2.9$ & 69.4 \\
\hline $75-85$ & 79.8 & $55.2 \pm 2.6$ & 53.1 \\
\hline $85-100$ & 92.2 & $38.8 \pm 2.2$ & 39.8 \\
\hline $100-115$ & 107.2 & $27.3 \pm 2.2$ & 29.4 \\
\hline $115-130$ & 122.3 & $20.0 \pm 2.1$ & 22.6 \\
\hline $130-145$ & 137.3 & $17.3 \pm 2.1$ & 17.9 \\
\hline $145-175$ & 159.3 & $9.1 \pm 2.0$ & 13.3 \\
\hline
\end{tabular}

The full L3 data sample at $\sqrt{s}=91.2-209.2 \mathrm{GeV}$ is considered and the cosine of the electron rest-frame scattering angle is limited to the range $\left|\cos \theta^{*}\right|<0.8$.

observed and expected events, also shown in Fig. $4 \mathrm{~b}$ and $\mathrm{c}$.

The differential cross section of the $\mathrm{e}^{+} \mathrm{e}^{-} \rightarrow$ $\mathrm{e}^{+} \mathrm{e}^{-} \gamma$ process as a function of $\sqrt{s^{\prime}}$ is related to the cross section of the $\gamma \mathrm{e}^{ \pm} \rightarrow \gamma \mathrm{e}^{ \pm}$process by:

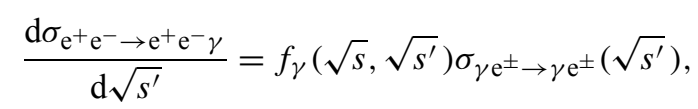



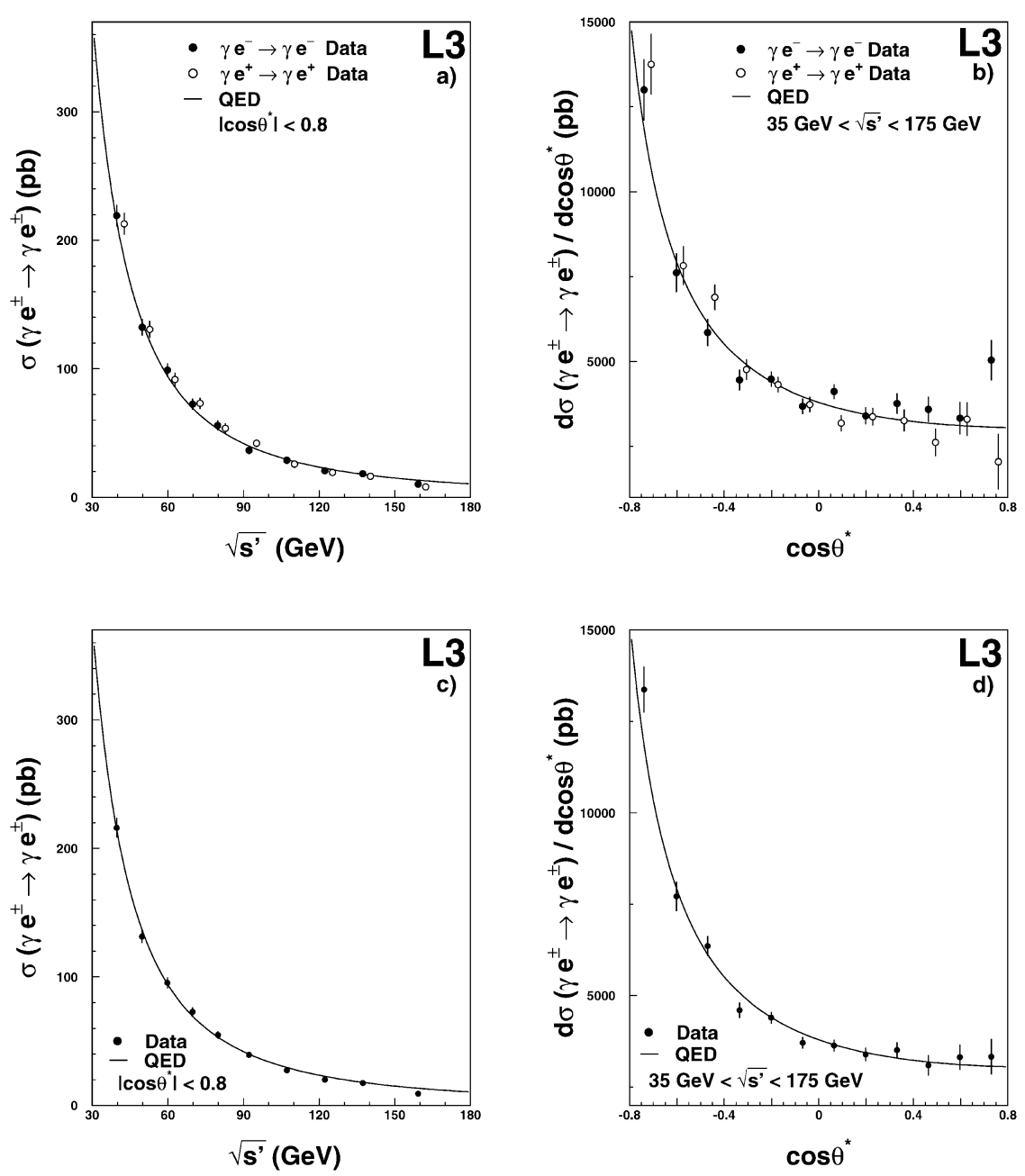

Fig. 6. Measured cross sections of the $\gamma \mathrm{e}^{+} \rightarrow \gamma \mathrm{e}^{+}, \gamma \mathrm{e}^{-} \rightarrow \gamma \mathrm{e}^{-}$and $\gamma \mathrm{e}^{ \pm} \rightarrow \gamma \mathrm{e}^{ \pm}$process as a function of (a) and (c) the effective centre-of-mass energy for $\left|\cos \theta^{*}\right|<0.8$ and (b) and (d) the rest-frame scattering angle for $\sqrt{s^{\prime}}=35-175 \mathrm{GeV}$. For clarity, the measurements for positrons in (a) and (b) are slightly displaced to the right. Data collected at $\mathrm{e}^{+} \mathrm{e}^{-}$centre-of-mass energies $\sqrt{s}=188.6-209.2 \mathrm{GeV}$ are considered and the QED predictions are also shown.

where $f_{\gamma}\left(\sqrt{s}, \sqrt{s^{\prime}}\right)$ is the virtual-photon flux. The cross section of the $\mathrm{e}^{+} \mathrm{e}^{-} \rightarrow \mathrm{e}^{+} \mathrm{e}^{-} \gamma$ process for the $i$ th interval of $\sqrt{s^{\prime}}$ can be extracted from the numbers of events observed in data and expected from signal and background Monte Carlo simulations, $N_{\text {data }}(i)$, $N_{\mathrm{MC}}^{\mathrm{sign}}(i)$ and $N_{\mathrm{MC}}^{\mathrm{back}}(i)$, respectively, as:

$$
\begin{aligned}
& \frac{\Delta \sigma_{\mathrm{e}^{+} \mathrm{e}^{-} \rightarrow \mathrm{e}^{+} \mathrm{e}^{-} \gamma}}{\Delta \sqrt{s^{\prime}}} \\
& =\frac{\Delta \sigma_{\mathrm{e}^{+} \mathrm{e}^{-} \rightarrow \mathrm{e}^{+} \mathrm{e}^{-} \gamma}^{\text {MC }} \frac{N_{\text {data }}(i)-N_{\mathrm{MC}}^{\text {back }}(i)}{\Delta \sqrt{s^{\prime}}},}{N_{\mathrm{MC}}^{\text {sign }}(i)}
\end{aligned}
$$

where $\Delta \sigma_{\mathrm{e}^{+} \mathrm{e}^{-} \rightarrow \mathrm{e}^{+} \mathrm{e}^{-} \gamma}^{\mathrm{MC}} / \Delta \sqrt{s^{\prime}}$ is the cross section predicted by the Monte Carlo. By expressing Eq. (3) in terms of Eq (2), and assuming that $f_{\gamma}\left(\sqrt{s}, \sqrt{s^{\prime}}\right)$ is the same for data and Monte Carlo, the cross section for quasi-real Compton scattering at the average effective centre-of-mass energy $\left\langle\sqrt{s^{\prime}}\right\rangle$ can be derived directly from the values in Table 3 as:

$$
\begin{aligned}
& \sigma_{\gamma \mathrm{e}^{ \pm} \rightarrow \gamma \mathrm{e}^{ \pm}}\left(\left\langle\sqrt{s^{\prime}}\right\rangle\right) \\
& =\sigma_{\gamma \mathrm{e}^{ \pm} \rightarrow \gamma \mathrm{e}^{ \pm}}^{\mathrm{QED}}\left(\left\langle\sqrt{s^{\prime}}\right\rangle\right) \frac{N_{\text {data }}(i)-N_{\mathrm{MC}}^{\mathrm{back}}(i)}{N_{\mathrm{MC}}^{\text {sign }}(i)},
\end{aligned}
$$




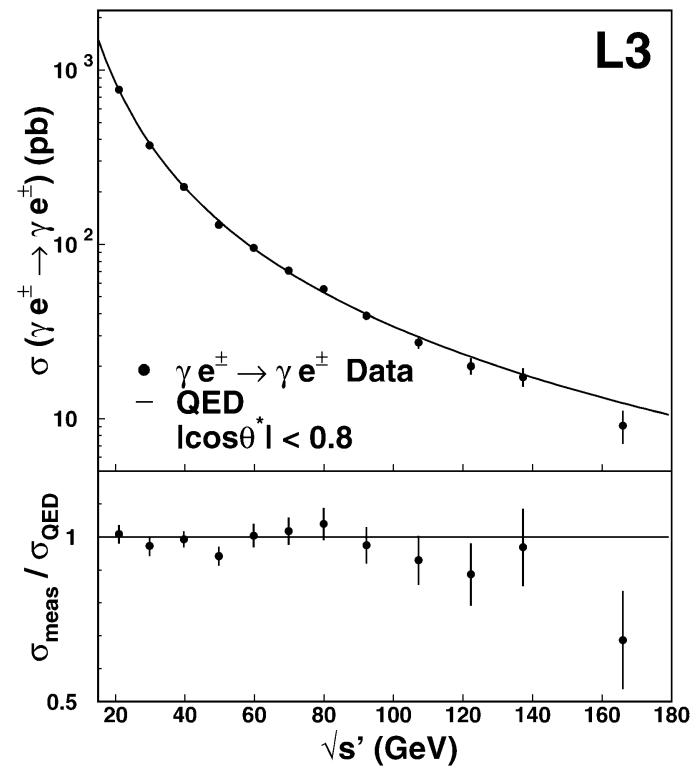

Fig. 7. Cross section of the $\gamma \mathrm{e}^{ \pm} \rightarrow \gamma \mathrm{e}^{ \pm}$process measured as a function of the effective centre-of-mass energy, $\sqrt{s^{\prime}}$, for $\left|\cos \theta^{*}\right|<0.8$, compared to the QED predictions. The full data sample collected at $\sqrt{s}=91.2-209.2 \mathrm{GeV}$ is considered and the cosine of the electron rest-frame scattering angle is limited to the range $\left|\cos \theta^{*}\right|<0.8$.

where $\sigma_{\gamma \mathrm{e}^{ \pm} \rightarrow \gamma \mathrm{e}^{ \pm}}^{\mathrm{QED}}\left(\left\langle\sqrt{s^{\prime}}\right\rangle\right)$ is the value expected from QED. The differential cross section for quasi-real Compton scattering as a function of $\cos \theta^{*}$ is derived from a formula equivalent to Eq. (4), mutatis mutandis.

The selected events are further classified according to the presence of either electrons or positrons in the final states. The cross section as a function of $\sqrt{s^{\prime}}$ and the differential cross section as a function of $\cos \theta$ are measured and the results are presented in Fig. $6 a$ and $b$. They are in good mutual agreement. The combined results for electrons and positrons are presented in Tables 3 and 4 and in Fig. 6c and d. All results are in good agreement with the QED predictions, also presented in Tables 3 and 4 and in Fig. 6. The predictions for the cross section as a function of $\sqrt{s^{\prime}}$ are derived by integrating Eq. (1) over the range $-0.8<\cos \theta^{*}<0.8$, while the predictions for the differential cross section as a function of $\cos \theta$ are derived by integrating over the range $35 \mathrm{GeV}<\sqrt{s^{\prime}}<$ $175 \mathrm{GeV}$.
The cross sections as a function of $\sqrt{s^{\prime}}$ measured in the range $\sqrt{s^{\prime}}=35-175 \mathrm{GeV}$ are combined with those L3 measured in the range $\sqrt{s^{\prime}}=20-100 \mathrm{GeV}$ [6]. The full data-sample collected by the L3 detector at $\sqrt{s}=91-209 \mathrm{GeV}$ is therefore considered, covering a range $\sqrt{s^{\prime}}=20-175 \mathrm{GeV}$. The results are presented in Table 5 and Fig. 7. They are in good agreement, over two orders of magnitude, with the QED predictions.

\section{References}

[1] ALEPH Collaboration, A. Heister, et al., Eur. Phys. J. C 28 (2003) 1;

DELPHI Collaboration, J. Abdallah, et al., Eur. Phys. J. C 37 (2004) 405;

L3 Collaboration, P. Achard, et al., Phys. Lett. B 531 (2002) 28 ;

OPAL Collaboration, G. Abbiendi, et al., Eur. Phys. J. C 26 (2003) 331.

[2] DELPHI Collaboration, J. Abdallah, et al., Eur. Phys. J. C 35 (2004) 159;

L3 Collaboration, P. Achard, et al., Phys. Lett. B 585 (2004) 53.

[3] F. Halzen, A.D. Martin, Quarks \& Leptons, Wiley, New York, 1984.

[4] C. Carimalo, P. Kessler, J. Parisi, Nucl. Phys. B 57 (1973) 582.

[5] G. Cosme, et al., Lett. Nuovo Cimento 8 (1973) 509.

[6] L3 Collaboration, M. Acciarri, et al., Phys. Lett. B 439 (1998) 183.

[7] L3 Collaboration, B. Adeva, et al., Nucl. Instrum. Methods A 289 (1990) 35;

L3 Collaboration, O. Adriani, et al., Phys. Rep. 236 (1993) 1; J.A. Bakken, et al., Nucl. Instrum. Methods A 275 (1989) 81; O. Adriani, et al., Nucl. Instrum. Methods A 302 (1991) 53;

B. Adeva, et al., Nucl. Instrum. Methods A 323 (1992) 109;

K. Deiters, et al., Nucl. Instrum. Methods A 323 (1992) 162; M. Acciarri, et al., Nucl. Instrum. Methods A 351 (1994) 300; G. Basti, et al., Nucl. Instrum. Methods A 374 (1996) 293.

[8] I.C. Brock, et al., Nucl. Instrum. Methods A 381 (1996) 236.

[9] M. Chemarin, et al., Nucl. Instrum. Methods A 349 (1994) 345.

[10] TEEGG version 7.1 is used; D. Karlen, Nucl. Phys. B 289 (1987) 23.

[11] A.S. Schmidt-Kärst, Elektron-Photon Physik bei LEP, Ph.D. thesis, RWTH Aachen, 2000.

[12] V.M. Budnev, et al., Phys. Rep. 15 (1975) 181.

[13] BHWIDE version 1.03 is used; S. Jadach, W. Placzek, B.F.L. Ward, Phys. Lett. B 390 (1997) 298.

[14] KK2f version 4.13 is used; S. Jadach, B.F.L. Ward, Z. Wạs, Comput. Phys. Commun. 130 (2000) 260.

[15] GGG Monte Carlo; F.A. Berends, R. Kleiss, Nucl. Phys. B 186 (1981) 22. 
[16] DIAG36 Monte Carlo;

F.A. Berends, P.H. Daverfeldt, R. Kleiss, Nucl. Phys. B 253 (1985) 441.

[17] GEANT version 3.15 is used;

R. Brun, et al., preprint CERN DD/EE/84-1, 1985, revised 1987.
[18] R. Vasquez, Study of single Z-boson production and Compton scattering in electron-positron collisions at LEP at centre-ofmass energies up to $209 \mathrm{GeV}$, Ph.D. thesis, Purdue University, 2005.

[19] G.D. Lafferty, T.R. Wyatt, Nucl. Instrum. Methods A 355 (1995) 541 